\title{
Resveratrol inhibits the phosphatidylinositide 3-kinase/protein kinase $B$ /mammalian target of rapamycin signaling pathway in the human chronic myeloid leukemia K562 cell line
}

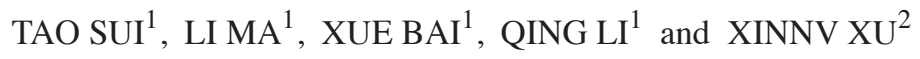 \\ ${ }^{1}$ Department of Hematology; ${ }^{2}$ Key Laboratory for Critical Care Medicine of the Ministry of Health, \\ Tianjin First Center Hospital, Tianjin 300192, P.R. China
}

Received September 15, 2013; Accepted March 14, 2014

DOI: $10.3892 / \mathrm{ol} .2014 .2014$

\begin{abstract}
Resveratrol inhibits the initiation, promotion and progression of tumors, however, the mechanism by which resveratrol inhibits the proliferation of the human chronic myeloid leukemia K562 cell line remains unclear. The present study was conducted to investigate the effect of resveratrol on the activation of the phosphatidylinositide 3-kinase (PI3K)/protein kinase B (Akt)/mammalian target of rapamycin (mTOR) signaling cascade in K562 cells. Resveratrol showed significant cytotoxic effects and induced apoptosis in K562 cells in a dose- and time-dependent manner. In addition, resveratrol attenuated the phosphorylation of PI3K, Akt and mTOR in the K562 cells. Furthermore, the selected inhibitors of PI3K (LY294002), Akt (SH-6) and mTOR (rapamycin) enhanced the effects of resveratrol in K562 cells. In addition, cyclin D1 levels were found to decrease and the activation of caspase-3 was observed. Resveratrol was also found to significantly attenuate the phosphorylation of the downstream molecules, p70S6K and 4EBP1. These results suggested that the downregulation of the PI3K/Akt/mTOR signaling cascades may be a crucial mediator in the inhibition of proliferation and induction of apoptosis by resveratrol in K562 cells.
\end{abstract}

\section{Introduction}

Chronic myeloid leukemia (CML) is a clonal myeloproliferative hematopoietic stem cell disease, characterized by the presence of the Philadelphia chromosome, which is generated by the reciprocal translocation of the ABL1 oncogene localized on chromosome 9 with the breakpoint

Correspondence to: Dr Tao Sui, Department of Hematology, Tianjin First Center Hospital, 24 Fukang Road, Tianjin 300192, P.R. China

E-mail: taosui71@163.com

Key words: phosphatidylinositide 3-kinase, protein kinase B, mammalian target of rapamycin, chronic myeloid leukemia, resveratrol cluster region (BCR) on chromosome 22 [t $(9 ; 22)](1,2)$. The BCL-ABL fusion gene, which has been identified as a crucial step in the pathogenesis of CML, encodes the fusion BCR-ABL1 protein, which possesses constitutive tyrosine kinase activity $(3,4)$. The BCR-ABL tyrosine kinase significantly influences several cellular signaling cascades, including the Ras, mitogen-activated protein kinase, phosphatidylinositide 3-kinase (PI3K)/protein kinase B (Akt), signal transducer and activator of transcription 5, and Src (5-9). Abnormal overactivation of these signaling cascades leads to increased cellular proliferation, resistance to apoptosis and genetic instability.

The selective tyrosine kinase inhibitors (TKI), which target the ATP binding site of BCR/ABL, block BCR/ABL kinase activity and exhibit a positive therapeutic effect for CML $(10,11)$. TKIs, such as imatinib, are considered as a conventional treatment option for CML. However, CML patients may become relatively resistant to TKI therapy, including the second generation TKIs $(12,13)$ and, thus, the development of a therapeutic strategy that targets abnormal signaling cascades other than $\mathrm{BCR} / \mathrm{ABL}$ is urgently required for CML treatment.

Resveratrol is a naturally occurring polyphenolic compound that is abundant in various plants, including grapes and nuts, as well as red wine and other plant-based food sources (14). Resveratrol has been reported to exert multiple beneficial effects, including anti-inflammatory, -oxidant and -viral effects, as well as neuroprotection $(15,16)$. Resveratrol inhibits the initiation, promotion and progression of tumors and, therefore, based on the above report, resveratrol may present a potential preventive and therapeutic agent (17). Numerous studies have demonstrated that resveratrol is involved in the regulation of cell cycle arrest, differentiation and apoptosis of different tumor cell lines and experimental cancer models (18-22).

Resveratrol causes cell cycle arrest at the G1 phase by inducing the expression of the cyclin-dependent kinase inhibitors, p21WAF1/CIP1 and p27KIP1 (23). Additionally, resveratrol induces cell apoptosis by upregulating the expression of Bax, Bak, Bim, p53, tumor necrosis factor-related apoptosis-inducing ligand (TRAIL), TRAIL-receptor (R)1/R4 and TRAIL-R2/R5, whilst 
simultaneously downregulating the expression of B-cell lymphoma (Bcl)-2 and Bcl-XL $(24,25)$. Numerous studies have shown that resveratrol modulates several cell signaling pathways, which are involved in the proliferation of tumor cells. Furthermore, resveratrol functions through different mechanisms in different types of tumor cells (26).

The aim of the present study was to investigate whether resveratrol has potential antitumor effects in human CML and to determine whether the modulation of the $\mathrm{PI} 3 \mathrm{~K} / \mathrm{Akt} / \mathrm{mammalian}$ target of rapamycin (mTOR) signaling pathway by resveratrol is crucial for its anticancer effects in the human CML K562 cell line.

\section{Materials and methods}

Reagents and antibodies. Resveratrol was purchased from Sigma-Aldrich (St. Louis, MO, USA) and dissolved in dimethylsulfoxide (Sigma-Aldrich) as a stock solution of $100 \mathrm{mM}$. Resveratrol was further diluted in RPMI-1640 medium (Gibco, Big Cabin, OK, USA) plus 10\% fetal bovine serum (FBS; Gibco) to the appropriate final concentrations. The primary polyclonal rabbit anti-human antibodies, anti-PI3K, -phosphorylated (p)-PI3K (Tyr458), -Akt, -p-Akt (Ser473), -mTOR, -p-mTOR (Ser2448), -p70S6K, -p-p70S6K (Thr389), -4EBP1, -p-4EBP1 (Ser65), -cyclin D1, -procaspase-3, -cleaved caspase-3 and- $\beta$-actin, were obtained from Cell Signaling Technology, Inc. (Beverly, MA, USA), and the secondary horseradish peroxidase (HRP)-labeled mouse anti-rabbit IgG polyclonal antibodies for western blot analysis were provided by Beijing Zhongshan Golden Bridge Biotechnology Co., Ltd. (Beijing, China). Annexin V-fluorescein isothiocyanate (FITC) and propidium iodide (PI) were purchased from BD Biosciences (Palo Alto, CA, USA), LY294002 and SH-6 were provided by Santa Cruz Biotechnology, Inc. (Dallas, TX, USA), and rapamycin (RAPA) was purchased from the North China Pharmaceutical Group Corporation (Shijiazhuang, China).

Cell culture. The human CML K562 cell line was purchased from the Peking Union Medical College Cell Library (Beijing, China). The cells were cultured in RPMI-1640 medium supplemented with $100 \mathrm{U} / \mathrm{ml}$ of penicillin, $100 \mu \mathrm{g}$ streptomycin (both Gibco) and $10 \% \mathrm{FBS}$ at $37^{\circ} \mathrm{C}$ in a humidified atmosphere containing $5 \% \mathrm{CO}_{2}$.

Cell Counting Kit-8 (CCK-8) assay. Cell proliferation was determined using water-soluble tetrazolium salt- 8 dye (Sigma-Aldrich) according to the manufacturer's instructions. Briefly, the cells were suspended in RPMI-1640 medium containing $10 \%$ FBS and seeded at a density of $5 \times 10^{3}$ cells/well in 96-well plates. Resveratrol was then added to the medium at various concentrations of up to $60 \mu \mathrm{M}$ for different time durations. Next, the CCK-8 solution (10 $\mu$ l; Sigma-Aldrich) was added to each well and further incubated at $37^{\circ} \mathrm{C}$ for $3 \mathrm{~h}$. The absorbance was determined at a wavelength of $450 \mathrm{~nm}$ using a microplate reader (Bio-Rad, Hercules, CA, USA). In total, three duplicate wells were set up for each experimental condition.

Apoptosis analysis. A total of $1 \times 10^{6}$ cells/well were treated with resveratrol for $24 \mathrm{~h}$ and double-staining with
Annexin V-FITC $(1 \mu \mathrm{l})$ and PI $(1 \mu \mathrm{g})$ was performed. The cells were then washed with phosphate-buffered saline (PBS; Beyotime, Haikou, China) and analyzed by flow cytometry (FACS sort; BD Biosciences).

Western blot analysis. Western blot analysis was performed on whole cell extracts obtained by the direct dissolution of cells using a whole cell protein extract reagent (M-PER; Pierce Biotechnology, Inc., Rockford, IL, USA) according to the manufacturer's instructions. Protein concentrations were then determined using a bicinchoninic acid protein assay kit and bovine serum albumin [both Sangon Biotech (Shanghai) Co,. Ltd., Shanghai, China] was used as a control. Next, the proteins (40 $\mu \mathrm{g} / \mathrm{lane})$ were separated on $12 \%$ SDS-PAGE gels (Beyotime) and transferred onto polyvinylidene difluoride membranes (Bio-Rad). The membranes were then blocked with 5\% non-fat milk in phospshate-buffered saline with Tween [PBST; 0.2\% Tween-20 in PBS (pH 7.6); Beyotime] and incubated with the primary antibodies $(1: 1,000)$ for $18-24 \mathrm{~h}$ at $4^{\circ} \mathrm{C}$. The membranes were subsequently incubated with the secondary antibodies conjugated to $\operatorname{HRP}(1: 5,000)$ for $1 \mathrm{~h}$ at $37^{\circ} \mathrm{C}$. Finally, the protein bands were visualized using an enhanced chemiluminescence western blot detection kit (Pierce Biotechnology, Inc.).

Statistical analysis. Data are presented as the mean \pm standard deviation and were analyzed using SPSS 16.0 software (SPSS, Inc., Chicago, IL, USA). Significant differences were determined using one-way analysis of variance or a two-tailed Student's t-test. $\mathrm{P}<0.05$ was considered to indicate a statistically significant difference.

\section{Results}

Resveratrol inhibits the proliferation and induces the apoptosis of $K 562$ cells. To investigate the effect of resveratrol on the proliferation of K562 cells, the cells were treated with resveratrol at serial concentrations and its ability to inhibit proliferation was measured using a CCK- 8 assay. The results showed that resveratrol may inhibit the proliferation of K562 cells in a dose- and time-dependent manner (Fig. 1A and B). To further investigate whether resveratrol can induce the apoptosis of K562 cells, the cells were treated with $60 \mu \mathrm{M}$ of resveratrol for $24 \mathrm{~h}$ and the apoptotic rate of the K562 cells was detected using Annexin V-FITC/PI staining (Fig. 1C and D). The results suggested that resveratrol inhibits cell proliferation and induces apoptosis in K562 human CML cells.

Resveratrol blocks PI3K/Akt phosphorylation in $K 562$ cells. The PI3K/Akt signaling pathway is important in cell proliferation, differentiation and survival and resveratrol has been demonstrated to inhibit proliferation and induce apoptosis in various types of cancer cells (27). In the present study, to investigate whether PI3K/Akt phosphorylation is responsible for resveratrol-induced inhibition of proliferation, the K562 cells were treated with various concentrations of resveratrol for $24 \mathrm{~h}$. The levels of p-PI3K and p-Akt were then detected and the results demonstrated that resveratrol significantly blocks the constitutive phosphorylation of PI3K at Tyr458 and Akt at Ser473 in a dose-dependent manner (Fig. 2A). The K562 
A

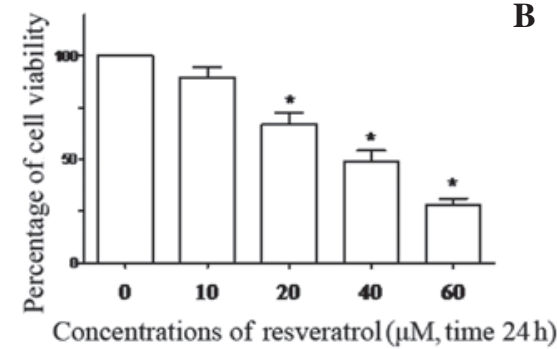

B

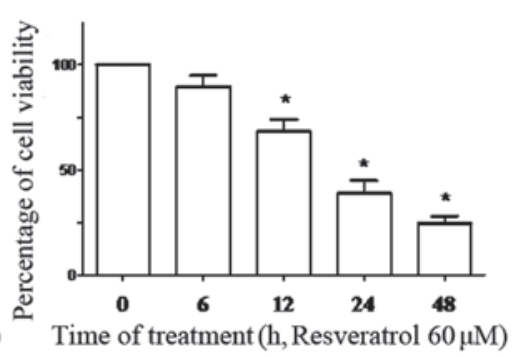

C

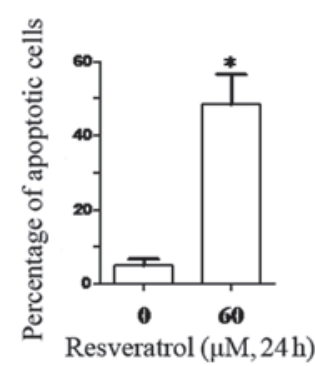

D

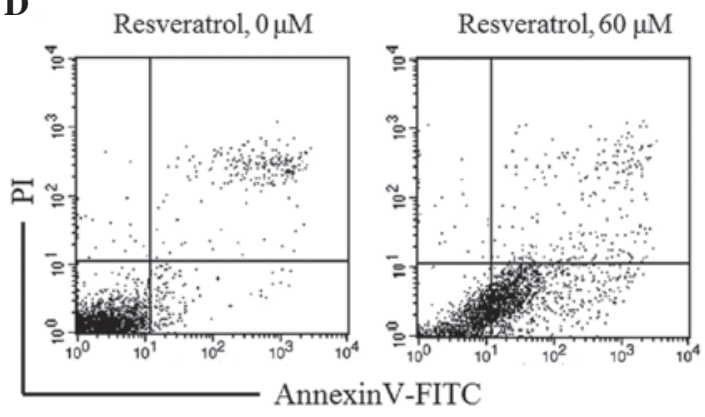

Figure 1. Resveratrol inhibits proliferation and induces apoptosis in K562 cells. Cells were treated at the indicated times and concentrations. The percentage of cell viability was determined by Cell Counting Kit-8 assay and resveratrol inhibited growth activity in a (A) dose- and (B) time-dependent manner. (C) Effect of resveratrol on apoptosis. (D) Double-staining with Annexin V-FITC and PI analyzed by flow cytometric following the exposure of cells to resveratrol for $24 \mathrm{~h}$ is presented as a representative plot of double staining with Annexin V-FITC and PI. Data are expressed as the mean \pm standard deviation of triplicate experiments."P $<0.05$ vs. $0 \mu \mathrm{M}$ resveratrol treatment. FITC, fluorescein isothiocyanate; PI, propidium iodide.

A

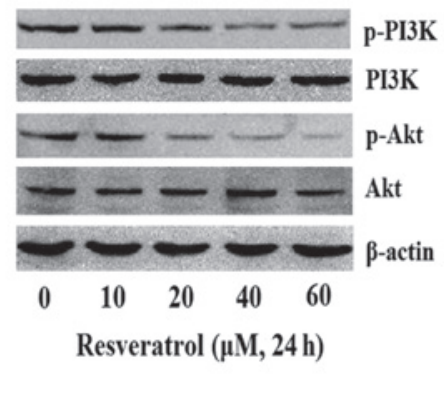

C

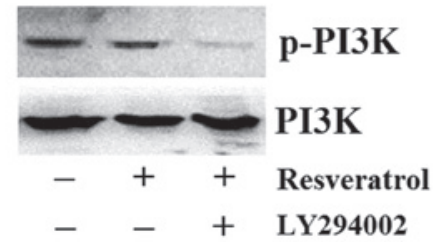

B

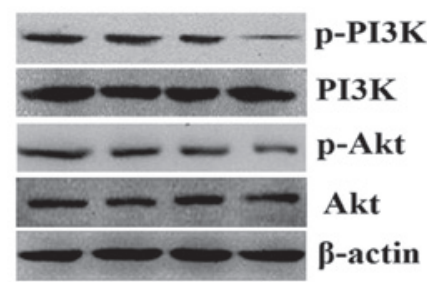

$\begin{array}{llll}0 & 6 & 12 & 24\end{array}$

Time (h, Resveratrol $60 \mu \mathrm{M})$

D

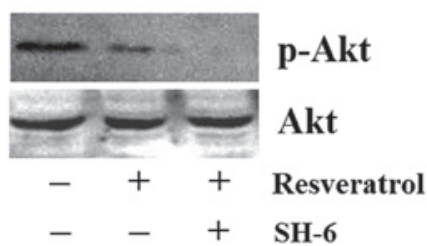

Figure 2. Resveratrol reduces the phosphorylation of PI3K and Akt in K562 cells. Levels of p-PI3K and p-Akt in K562 cells treated with (A) various concentrations of resveratrol for $24 \mathrm{~h}$ and (B) $60 \mu \mathrm{M}$ of resveratrol for different time periods, as determined by western blot analysis. (C) The selective inhibitor, LY294002, enhanced the resveratrol effect on reducing the phosphorylation of PI3K and (D) the selective inhibitor, SH-6, enhanced the resveratrol effect on reducing the phosphorylation of Akt. $\beta$-actin was used as a loading control for PI3K and Akt, while PI3K and Akt were used as loading controls for p-PI3K and Akt, respectively. PI3K, phosphatidylinositide 3-kinase; p-PI3K, phosphorylated-PI3K; Akt, protein kinase B; p-Akt, phosphorylated-Akt.

cells were then treated with $60 \mu \mathrm{M}$ of resveratrol for $0,6,12$ and $24 \mathrm{~h}$, and the levels of p-PI3K and p-Akt were observed to decrease following $24 \mathrm{~h}$ of treatment (Fig. 2B). The K562 cells were also treated with the selective PI3K inhibitor, LY294002, or the selective Akt inhibitor, SH-6. The selective inhibitors were observed to further inhibit the phosphorylation of PI3K and Akt in K562 cells induced by resveratrol, respectively (Fig. 2C). These results indicated that the antiproliferative effects of resveratrol in K562 cells are associated with blocking the activation of the PI3K/Akt signaling cascade.
Resveratrol suppresses the phosphorylation of mTOR in K562 cells. The mTOR protein is one of the downstream targets of PI3K/Akt. To investigate the effect of resveratrol on mTOR phosphorylation, the K562 cells were treated with a serial concentration of resveratrol for $24 \mathrm{~h}$ and resveratrol was found to reduce the levels of p-mTOR (Ser2448) in a dose-dependent manner (Fig. 3A). The K562 cells were then treated with $60 \mu \mathrm{M}$ of resveratrol for $0,6,12$ and $24 \mathrm{~h}$ and the level of p-mTOR was observed to decrease following resveratrol treatment for $12 \mathrm{~h}$ (Fig. 3B), and was further reduced 


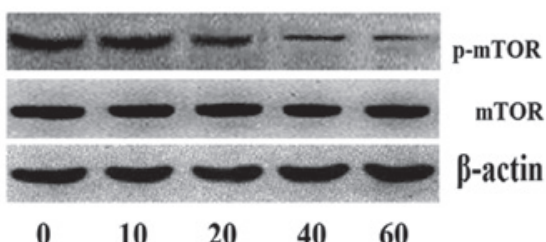

Resveratrol $(\mu \mathrm{M}$, time 24 h)

C

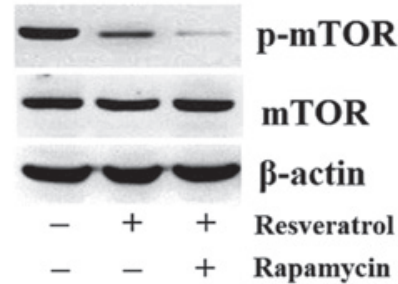

B

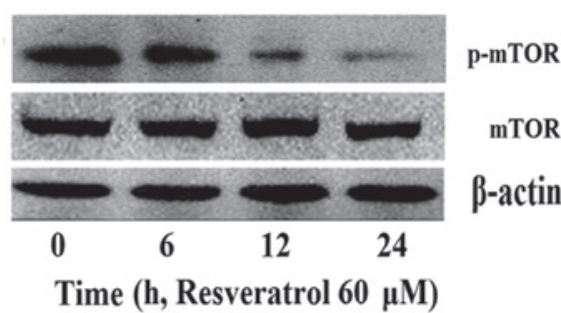

D

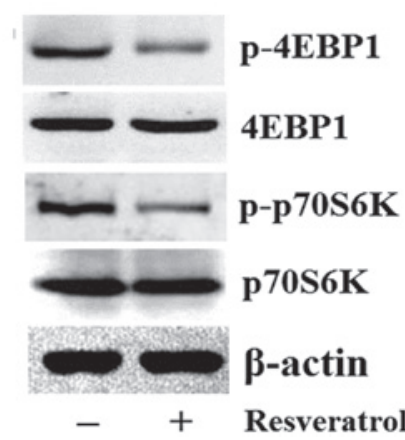

Figure 3. Resveratrol suppresses the phosphorylation of mTOR and its downstream targets in K562 cells. Levels of p-mTOR in K562 cells treated with (A) various concentrations of resveratrol for $24 \mathrm{~h}$ (which were then lysed) and (B) $60 \mu \mathrm{M}$ of resveratrol for different time periods, as determined by western blot analysis. (C) Inhibitor rapamycin enhanced the resveratrol effect on reducing the phosphorylation of mTOR and (D) resveratrol reduced the phosphorylation of p70S6K and 4EBP1. $\beta$-actin was used as a loading control for mTOR, 4EBP1 and p70S6k, while mTOR, 4EBP1 and p70S6k were used as loading controls for p-mTOR, p-4EBP1 and p-p70S6K, respectively. mTOR, mammalian target of rapamycin; p-mTOR, phosphorylated-mTOR.

A

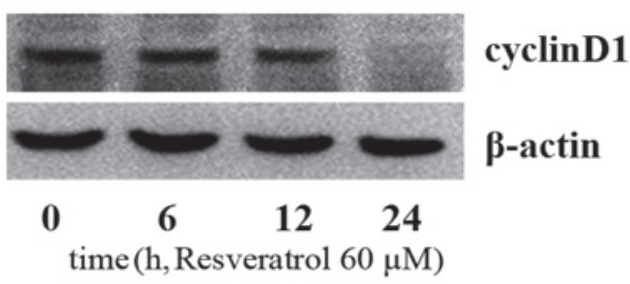

B

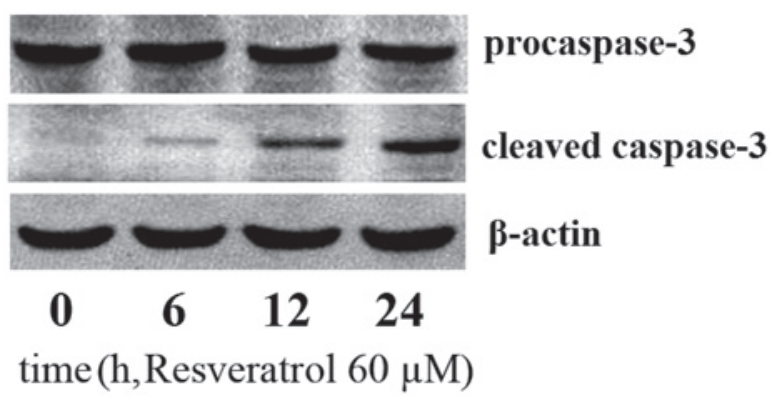

Figure 4. Resveratrol decreases cyclin D1 and increases caspase-3 expression. The expression of (A) cyclin D1 and (B) pro- and cleaved-caspase-3 was detected by western blot analysis in K562 cells which were treated with $60 \mu \mathrm{M}$ of resveratrol for different time periods. $\beta$-actin was used as a loading control for cyclin D1 and procaspase-3, while procaspase-3 was used as a loading control for cleaved caspase-3.

following rapamycin treatment (Fig. 3C). These results indicated that resveratrol inhibits the PI3K/Akt/mTOR signaling pathway and that the specific inhibitor of mTOR enhances the effect induced by resveratrol.

Next, the effect of resveratrol on the downstream targets of mTOR was investigated. The K562 cells were treated with 0 or $60 \mu \mathrm{M}$ of resveratrol for $24 \mathrm{~h}$ and the downstream targets were detected using western blot analysis. The results revealed that treatment with resveratrol reduced the phosphorylation of the downstream targets, p70S6K and 4EBP1; however, total p706SK and 4EBP1 levels were not affected by resveratrol treatment (Fig. 3D), which indicated that resveratrol may downregulate the Akt/mTOR signaling pathway.

Resveratrol suppresses cyclin D1 and enhances caspase-3 expression. Constitutive activation of the PI3K/Akt/mTOR signaling pathway has been demonstrated to exhibit a critical function in the cell cycle and antiapoptosis by affecting several regulatory molecules, including the upregulation of cyclin D1 and downregulation of caspase-3 expression (28). In the current study, to investigate whether resveratrol downregulates cyclin D1 expression and upregulates caspase-3 expression, the K562 cells were treated with $60 \mu \mathrm{M}$ of resveratrol. A marked decline in cyclin D1 levels and an increase in caspase-3 levels in the resveratrol-treated cells was observed (Fig. 4).

\section{Discussion}

Although resveratrol has been reported to have a wide range of potential targets during the inhibition of proliferation and induction of apoptosis in a variety tumor cell types (28), the underlying molecular mechanisms of its anticancer effects are not well understood, particularly in human leukemia which often proves difficult to treat as multiple signaling pathways may be involved (17). Contradictory results have previously been reported with regard to the inhibition of proliferation and induction of apoptosis by resveratrol. Certain studies have reported that resveratrol treatment induces apoptosis in various tumor cells (29-33). However, other studies have reported that resveratrol induces differentiation, but not apoptosis, in certain types of cancer cells (34-36). In the current study, one of the possible mechanisms of resveratrol-induced apoptosis of human CML K562 cells, was investigated. 
There is much evidence to support the critical function of the PI3K/Akt/mTOR signaling pathway in cancer proliferation, tumor genesis and metastasis $(37,38)$, and numerous studies have demonstrated that PI3K/Akt/mTOR activity is increased in a variety of tumor cell lines (39-42), including leukemia (43). In addition, it has been demonstrated that the inhibition of PI3K/Akt activation or expression may inhibit cancer cell proliferation and invasion. This signaling pathway involves three key driving proteins, PI3K, Akt and mTOR. The PI3K proteins are a family of lipid kinases, which include PI3K1, PI3K2 and PI3K3. Akt belongs to a family of serine/threonine protein kinases, which can be activated in a PI3K-dependent manner following stimulation by growth factors, stress or protein phosphatases. Furthermore, mTOR is one of the main downstream target molecules of PI3K/Akt, which performs a crucial function in the regulation of proliferation, differentiation and survival of cells. It has been demonstrated that the activation of the PI3K/Akt/mTOR signaling pathway increases the phosphorylation of p70S6K and 4EBP1, and that p-p70S6K subsequently induces the phosphorylation of the ribosomal protein $\mathrm{S} 6$.

The effect of resveratrol on the PI3K/Akt/mTOR pathway in CML cells has not been widely investigated. The results of the current study revealed that resveratrol inhibits the proliferation and induces the apoptosis of K562 cells in a dose-dependent manner. In addition, resveratrol downregulates the PI3K/Akt/mTOR signaling pathway and the inhibitors of the proteins involved in this signaling pathway enhance the inhibitory effects induced by resveratrol in K562 cells. Furthermore, resveratrol inhibits proliferation and induces apoptosis in human leukemia K562 cells, which is considered to occur via the deregulation of the cell cycle machinery and activation of mitochondria-mediated caspase-3 dependent apoptotic signaling cascades. These results indicated that resveratrol is an attractive candidate for use in leukemia therapy. Therefore, further understanding of the underlying molecular signaling mechanisms of the inhibition of proliferation and induction of CML cell apoptosis induced by resveratrol may aid the development of additional therapeutic targets for the treatment of CML.

Resveratrol inhibits the proliferation and induces the apoptosis of cells mediated by the regulation of cell cycle, proapoptotic and antiapoptotic proteins, such as cyclin D1 and caspase-3. Gao et al (44) demonstrated that the PI3K/Akt/mTOR signaling pathway exhibits a key function in the cell cycle progression in human prostate cancer cells. In addition, resveratrol has been demonstrated to inhibit the proliferation and induce the apoptosis of cells by downregulation of the nuclear factor- $\kappa \beta$ signaling pathway (45).

In conclusion, the present study demonstrated that resveratrol downregulates and inactivates the PI $3 \mathrm{~K} / \mathrm{Akt} / \mathrm{mTOR}$ signaling pathway, which may exhibit a critical function in resveratrol-induced apoptosis in K562 cells and, therefore, the PI3K/Akt/mTOR signaling pathway may present a potential therapeutic target for the treatment of human CML.

\section{Acknowledgements}

The present study was supported by grants from the Tianjin Science and Technology Committee (grant no. 11JCZDJC18600) and the Tianjin Municipal Health Bureau (grant no. 13KG106).

\section{References}

1. Chandra HS, Heisterkamp NC, Hungerford A, Morrissette JJ, Nowell PC, Rowley JD and Testa JR: Philadelphia Chromosome Symposium: commemoration of the 50th anniversary of the discovery of the Ph chromosome. Cancer Genet 204: 171-179, 2011.

2. Deininger MW, Goldman JM and Melo JV: The molecular biology of chronic myeloid leukemia. Blood 96: 3343-3356, 2000.

3. Hantschel $\mathrm{O}$ and Superti-Furga G: Regulation of the c-Abl and Bcr-Abl tyrosine kinases. Nat Rev Mol Cell Biol 5: 33-44, 2004.

4. Sattler M and Griffin JD: Molecular mechanisms of transformation by the BCR-ABL oncogene. Semin Hematol 40 (Suppl 2): S4-S10, 2003.

5. Barrett D, Brown VI, Grupp SA and Teachey DT: Targeting the $\mathrm{PI} 3 \mathrm{~K} / \mathrm{AKT} / \mathrm{mTOR}$ signaling axis in children with hematologic malignancies. Paediatr Drugs 14: 299-316, 2012.

6. Horita M, Andreu EJ, Benito A, Arbona C, Sanz C, Benet I, et al: Blockade of the Bcr-Abl kinase activity induces apoptosis of chronic myelogenous leukemia cells by suppressing signal transducer and activator of transcription 5-dependent expression of Bcl-xL. J Exp Med 191: 977-984, 2000.

7. Pene-Dumitrescu T and Smithgall TE: Expression of a Src family kinase in chronic myelogenous leukemia cells induces resistance to imatinib in a kinase-dependent manner. J Biol Chem 285: 21446-21457, 2010.

8. Baum KJ and Ren R: Effect of Ras inhibition in hematopoiesis and BCR/ABL leukemogenesis. J Hematol Oncol 1: 5, 2008.

9. Notari M, Neviani P, Santhanam R, Blaser BW, Chang JS, Galietta A, et al: A MAPK/HNRPK pathway controls BCR/ABL oncogenic potential by regulating MYC mRNA translation. Blood 107: 2507-2516, 2006.

10. Baran Y and Saydam G: Cumulative clinical experience from a decade of use: imatinib as first-line treatment of chronic myeloid leukemia. J Blood Med 3: 139-150, 2012.

11. Mauro MJ and Deininger MW: Chronic myeloid leukemia in 2006: a perspective. Haematologica 91: 152, 2006.

12. Shah NP and Sawyers CL: Mechanisms of resistance to STI571 in Philadelphia chromosome-associated leukemias. Oncogene 22: 7389-7395, 2003.

13. Sawyers CL, Hochhaus A, Feldman E, Goldman JM, Miller CB, Ottmann OG, et al: Imatinib induces hematologic and cytogenetic responses in patients with chronic myelogenous leukemia in myeloid blast crisis: results of a phase II study. Blood 99: 3530-3539, 2002 .

14. Soleas GJ, Diamandis EP and Goldberg DM: Resveratrol: a molecule whose time has come? And gone? Clin Biochem 30: 91-113, 1997.

15. Jang DS, Kang BS, Ryu SY, Chang IM, Min KR and Kim Y: Inhibitory effects of resveratrol analogs on unopsonized zymosan-induced oxygen radical production. Biochem Pharmacol 57: 705-712, 1999.

16. Inoue H, Jiang XF, Katayama T, Osada S, Umesono K and Namura S: Brain protection by resveratrol and fenofibrate against stroke requires peroxisome proliferator-activated receptor alpha in mice. Neurosci Lett 352: 203-206, 2003.

17. Yu W, Fu YC and Wang W: Cellular and molecular effects of resveratrol in health and disease. J Cell Biochem 113: 752-759, 2012.

18. Delmas D, Solary E and Latruffe N: Resveratrol, a phy tochemical inducer of multiple cell death pathways: apoptosis, autophagy and mitotic catastrophe. Curr Med Chem 18: 1100-1121, 2011.

19. Tinhofer I, Bernhard D, Senfter M, Anether G, Loeffler M, Kroemer G, et al: Resveratrol, a tumor-suppressive compound from grapes, induces apoptosis via a novel mitochondrial pathway controlled by Bcl-2. FASEB J 15: 1613-1615, 2001.

20. Mgbonyebi OP, Russo J and Russo IH: Antiproliferative effect of synthetic resveratrol on human breast epithelial cells. Int $\mathbf{J}$ Oncol 12: 865-869, 1998.

21. Ahmad N, Adhami VM, Afaq F, Feyes DK and Mukhtar H: Resveratrol causes WAF-1/p21-mediated G(1)-phase arrest of cell cycle and induction of apoptosis in human epidermoid carcinoma A431 cells. Clin Cancer Res 7: 1466-1473, 2001.

22. Dörrie J, Gerauer H, Wachter Y and Zunino SJ: Resveratrol induces extensive apoptosis by depolarizing mitochondrial membranes and activating caspase-9 in acute lymphoblastic leukemia cells. Cancer Res 61: 4731-4739, 2001.

23. Benitez DA, Pozo-Guisado E, Alvarez-Barrientos A, Fernandez-Salguero PM and Castellón EA: Mechanisms involved in resveratrol-induced apoptosis and cell cycle arrest in prostate cancer-derived cell lines. J Androl 28: 282-293, 2007. 
24. Fulda S and Debatin KM: Resveratrol-mediated sensitisation to TRAIL-induced apoptosis depends on death receptor and mitochondrial signalling. Eur J Cancer 41: 786-798, 2005.

25. Shankar S, Siddiqui I and Srivastava RK: Molecular mechanisms of resveratrol (3,4,5-trihydroxy-trans-stilbene) and its interaction with TNF-related apoptosis inducing ligand (TRAIL) in androgen-insensitive prostate cancer cells. Mol Cell Biochem 304: 273-285, 2007.

26. Shankar S, Singh G and Srivastava RK: Chemoprevention by resveratrol: molecular mechanisms and therapeutic potential. Front Biosci 12: 4839-4854, 2007.

27. Jiang H, Zhang L, Kuo J, Kuo K, Gautam SC, Croc L, et al: Resveratrol-induced apoptotic death in human U251 glioma cells. Mol Cancer Ther 4: 554-561, 2005.

28. Jiang H, Shang X, Wu H, Gautam SC, Al-Holou S, Li C, et al: Resveratrol downregulates PI3K/Akt/mTOR signaling pathways in human U251 glioma cells. J Exp Ther Oncol 8: 25-33, 2009.

29. Yang CS, Landau JM, Huang MT and Newmark HL: Inhibition of carcinogenesis by dietary polyphenolic compounds. Annu Rev Nutr 21: 381-406, 2001.

30. Quoc Trung L, Espinoza JL, Takami A and Nakao S: Resveratrol induces cell cycle arrest and apoptosis in malignant NK cells via JAK2/STAT3 pathway inhibition. PLoS One 8: e55183, 2013.

31. Miki H, Uehara N, Kimura A, Sasaki T, Yuri T, Yoshizawa K and Tsubura A: Resveratrol induces apoptosis via ROS-triggered autophagy in human colon cancer cells. Int J Oncol 40 : 1020-1028, 2012.

32. Hussain AR, Uddin S, Bu R, Khan OS, Ahmed SO, Ahmed M and Al-Kuraya KS: Resveratrol suppresses constitutive activation of AKT via generation of ROS and induces apoptosis in diffuse large B cell lymphoma cell lines. PLoS One 6: e24703, 2011.

33. Truong M, Cook MR, Pinchot SN, Kunnimalaiyaan M and Chen H: Resveratrol induces Notch2-mediated apoptosis and suppression of neuroendocrine markers in medullary thyroid cancer. Ann Surg Oncol 18: 1506-1511, 2011.

34. Chen Q, Ganapathy S, Singh KP, Shankar S and Srivastava RK: Resveratrol induces growth arrest and apoptosis through activation of FOXO transcription factors in prostate cancer cells. PLoS One 5: e15288, 2010
35. Leong CW, Wong CH, Lao SC, Leong EC, Lao IF, Law PT, et al: Effect of resveratrol on proliferation and differentiation of embryonic cardiomyoblasts. Biochem Biophys Res Commun 360: 173-180, 2007.

36. Kaminski J, Lançon A, Aires V, Limagne E, Tili E, Michaille JJ and Latruffe N: Resveratrol initiates differentiation of mouse skeletal muscle-derived C2C12 myoblasts. Biochem Pharmacol 84: 1251-1259, 2012.

37. Yu XM, Jaskula-Sztul R, Ahmed K, Harrison AD, Kunnimalaiyaan $\mathrm{M}$ and Chen $\mathrm{H}$ : Resveratrol induces differentiation markers expression in anaplastic thyroid carcinoma via activation of Notch1 signaling and suppresses cell growth. Mol Cancer Ther 12: 1276-1287, 2013.

38. Yap TA, Garrett MD, Walton MI, Raynaud F, de Bono JS and Workman P: Targeting the PI3K-AKT-mTOR pathway: progress, pitfalls, and promises. Curr Opin Pharmacol 8: 393-412, 2008.

39. Osaki M, Oshimura M and Ito H: PI3K-Akt pathway: its functions and alterations in human cancer. Apoptosis 9: 667-676, 2004

40. Wetzker R and Rommel C: Phosphoinositide 3-kinases as targets for therapeutic intervention. Curr Pharm Des 10: 1915-1922, 2004.

41. Fresno Vara JA, Casado E, de Castro J, Cejas P, Belda-Iniesta C and González-Barón M: PI3K/Akt signalling pathway and cancer. Cancer Treat Rev 30: 193-204, 2004.

42. Franke TF, Hornik CP, Segev L, Shostak GA and Sugimoto C: PI3K/Akt and apoptosis: size matters. Oncogene 22: 8983-8998, 2003.

43. Morgensztern D and McLeod HL: PI3K/Akt/mTOR pathway as a target for cancer therapy. Anticancer Drugs 16: 797-803, 2005.

44. Martelli AM,Evangelisti C, Chiarini F, Grimaldi C, Manzoli L and McCubrey JA: Targeting the PI3K/AKT/mTOR signaling network in acute myelogenous leukemia. Expert Opin Investig Drugs 18: 1333-1349, 2009.

45. Gao N, Zhang Z, Jiang BH and Shi X: Role of PI3K/AKT/mTOR signaling in the cell cycle progression of human prostate cancer. Biochem Biophys Res Commun 310: 1124-1132, 2003.

46. Sun C, Hu Y, Liu X, Wu T, Wang Y, He W and Wei W: Resveratro downregulates the constitutional activation of nuclear factor-kappaB in multiple myeloma cells, leading to suppression of proliferation and invasion, arrest of cell cycle, and induction of apoptosis. Cancer Genet Cytogenet 165: 9-19, 2006. 